MATEMATIKA, 2017, Volume 33, Number 2, 207-214

(C) Penerbit UTM Press. All rights reserved

\title{
Some new properties of Lucas-balancing and Lucas-cobalancing number
}

\author{
Shekh Mohammed Zahid \\ Institute of Mathematics and Applications \\ Bhubaneswar, Andharua, Odisha 751003, India \\ e-mail: shekhmohammedzahid@gmail.com
}

\begin{abstract}
In recent year Behera and Panda introduced a new number sequence that is solutions of the Diophantine equation $1+2+3 \ldots+(n-1)=(n+1)+(n+2) \ldots+$ $(n+r)$, where $n$ and $r$ are positive integers. If the pairs $(n, r)$ constitutes a solution of above equation then $n$ is called balancing number and $r$ is the corresponding balancer. The concept of balancing number is extended by introducing the notion of cobalancing which solution of the Diophantine equation $1+2+3 \ldots+N=$ $(N+1)+(N+2) \cdots+(N+R)$, where $N$ is called cobalancing number and $R$ is called corresponding cobalancer. Further, Panda introduced the concepts of corresponding Lucas-balancing defined as $C_{n}=\sqrt{8 B_{n}^{2}+1}$ and Lucas-cobalancing as $c_{n}=\sqrt{8 b_{n}^{2}+8 b_{n}+1}$, where $B_{n}$ is $n^{\text {th }}$ balancing number and $b_{n}$ is $n^{\text {th }}$ cobalancing number. In this paper, we investigate some new properties of Lucas-balancing and Lucas-cobalancing.
\end{abstract}

Keywords Diophantine equation; Balancing numbers; Cobalancing number; Lucasbalancing numbers; Lucas-cobalancing numbers

AMS mathematics subject classification 11B83, 11B37

\section{Introduction}

The concept of balancing number is introduced by Behera and Panda [1] in connection with Diophantine equation

$$
1+2+3 \ldots+(n-1)=(n+1)+(n+2)+\cdots+(n+r)
$$

for some $r \in Z^{+}$. Here $r$ is called the balancer corresponding to balancing number $n$. For example, 6 is balancing number with balancer 2. Balancing number follow recurrence relation $B_{n+1}=6 B_{n}-B_{n-1}$. Properties of balancing number are very similar to Fibonacci numbers. The study of balancing number and cobalancing number can be seen in [2].

Panda and Ray [3] modified the notion of balancing number to cobalancing number, in which natural $N$ is called cobalancing number if

$$
1+2+3 \ldots+N=(N+1)+(N+2)+\cdots+(N+R)
$$

for some natural number $R$, where $R$ is called cobalancer of cobalancing number $N$. The first three cobalancing numbers are 2, 14 and 84 with cobalancers 1,6 and 35 respectively.

Panda [4] introduced the Lucas-balancing number as $C_{n}=\sqrt{8 B_{n}^{2}+1}$, the first three Lucas-balancing number are 3,17 and 99. Further, he defined Lucas- cobalancing number as $c_{n}=\sqrt{8 b_{n}^{2}+8 b_{n}+1}$, first three Lucas-cobalancing are 1,7 and 40 , where $n \in Z^{+}$.

The Binet formula of balancing number, Lucas-balancing number and Lucas-cobalancing number are $B_{n}=\frac{\alpha_{1}^{2 n}-\alpha_{2}^{2 n}}{4 \sqrt{2}}, C_{n}=\frac{\alpha_{1}^{2 n}+\alpha_{2}^{2 n}}{2}$ and $c_{n}=\frac{\alpha_{1}^{2 n-1}+\alpha_{2}^{2 n-1}}{2}$, respectively, where $\alpha_{1}=$ $1+\sqrt{2}$ and $\alpha_{2}=1-\sqrt{2}$ (see $\left.[4,5]\right)$. 
In this paper, we investigate the properties of Lucas-balancing number and Lucascobalancing number. In this investigation we find the generating function of both sequences and their relation with balancing number.

Throughout the whole paper the balancing number starts from $B_{1}=1$ and $B_{2}=6$, Lucas-balancing number starts from $C_{1}=3$ and $C_{2}=17$ and Lucas-cobalancing number starts from $c_{1}=1$ and $c_{2}=7$.

\section{Generating function of Lucas-balancing and Lucas- cobalancing sequence}

In this section, we introduce generating function of Lucas-balancing and Lucas-cobalancing numbers. We use Lockwood [6] identity which expands $x^{n}+y^{n}$ and applying it to Binet form of the given sequence,

$$
x^{n}+y^{n}=(x+y)^{n}+\sum_{k=1}^{\lfloor n / 2\rfloor}(-1)^{k}\left[\left(\begin{array}{l}
n-k \\
k
\end{array}\right)+\left(\begin{array}{l}
n-k-1 \\
k-1
\end{array}\right)\right](x y)^{k}(x+y)^{n-2 k}
$$

where $\lfloor m\rfloor$ denotes the floor of real number $m$, that is, greatest integer less than or equal to $m$.

Theorem 2.1 The generating function of Lucas-balancing number is

$$
\sum_{k=0}^{n}\left[\left(\begin{array}{l}
2 n-k \\
k
\end{array}\right)+\left(\begin{array}{l}
2 n-k-1 \\
k-1
\end{array}\right)\right] 2^{2 n-2 k-1}
$$

where we define $\left(\begin{array}{c}r \\ -1\end{array}\right)=0$.

Proof Considering the Binet form of Lucas-balancing number and simplifying it using (3), we get the generating function of Lucas-balancing number as

$$
\begin{gathered}
C_{n}=\frac{2^{2 n}+\sum_{k=1}^{n}(-1)^{k}\left[\left(\begin{array}{l}
2 n-k \\
k
\end{array}\right)+\left(\begin{array}{l}
2 n-k-1 \\
k-1
\end{array}\right)\right](-1)^{k} 2^{2 n-2 k}}{2} \\
=\sum_{k=0}^{n}\left[\left(\begin{array}{l}
2 n-k \\
k
\end{array}\right)+\left(\begin{array}{l}
2 n-k-1 \\
k-1
\end{array}\right)\right] 2^{2 n-2 k-1}
\end{gathered}
$$

where we define $\left(\begin{array}{l}r \\ -1\end{array}\right)=0$. This proved the theorem.

Theorem 2.2 The generating function of Lucas-cobalancing number is

$$
c_{n}=\sum_{k=0}^{n-1}\left[\left(\begin{array}{l}
(2 n-1)-k \\
k
\end{array}\right)+\left(\begin{array}{l}
(2 n-1)-k-1 \\
k-1
\end{array}\right)\right] 2^{2 n-2 k-2},
$$

where we define $\left(\begin{array}{l}r \\ -1\end{array}\right)=0$. 
Proof Considering the Binet form of Lucas-cobalancing number and again simplifying it using (3), we get the generating function of Lucas-cobalancing number as

$$
c_{n}=\sum_{k=0}^{n-1}\left[\left(\begin{array}{l}
(2 n-1)-k \\
k
\end{array}\right)+\left(\begin{array}{l}
(2 n-1)-k-1 \\
k-1
\end{array}\right)\right] 2^{2 n-2 k-2}
$$

where we define $\left(\begin{array}{c}r \\ -1\end{array}\right)=0$. This proved the theorem.

\section{Connection between balancing, Lucas-balancing and Lucas- cobal- ancing sequence}

In this section, we give an important theorem that links Lucas-balancing and Lucascobalancing through a recurrence relation and later, we introduce some of its properties. Throughout this section we have $\alpha_{1}=1+\sqrt{2}, \alpha_{2}=1-\sqrt{2}$ and $\alpha_{1} \alpha_{2}=-1$.

Rationalizing $\alpha_{1}$, we get

$$
\begin{aligned}
\alpha_{1}=1+\sqrt{2} & =(1+\sqrt{2})\left(\frac{1-\sqrt{2}}{1-\sqrt{2}}\right) \\
& =\frac{-1}{1-\sqrt{2}}=-\alpha_{2}^{-1} .
\end{aligned}
$$

Theorem 3.1 The sequence of Lucas-balancing and Lucas-cobalancing satisfy recurrence relation $C_{n}-C_{n-1}=2 c_{n}$, where $n \in N$.

Proof Considering Binet form of Lucas-balancing and Lucas-cobalancing number and simplifying $C_{n}-C_{n-1}$,

$$
C_{n}-C_{n-1}=\frac{\alpha_{1}^{2 n}+\alpha_{2}^{2 n}}{2}-\frac{\alpha_{1}^{2 n-2}+\alpha_{2}^{2 n-2}}{2} .
$$

Since $\alpha_{1}=-\alpha_{2}^{-1}$ we simplify above equation as follows,

$$
\begin{aligned}
\frac{\alpha_{1}^{2 n}\left(1-\alpha_{1}^{-2}\right)+\alpha_{2}^{2 n}\left(1-\alpha_{2}^{-2}\right)}{2} & =\frac{\alpha_{1}^{2 n}\left(1-(1-\sqrt{2})^{2}\right)+\alpha_{2}^{2 n}\left(1-(1+\sqrt{2})^{2}\right)}{2} \\
& =\frac{-2 \alpha_{1}^{2 n}(1-\sqrt{2})-2 \alpha_{2}^{2 n}(1+\sqrt{2})}{2} \\
& =\frac{-2 \alpha_{1}^{2 n}\left(-\alpha_{1}^{-1}\right)-2 \alpha_{2}^{2 n}\left(-\alpha_{2}^{-1}\right)}{2} \\
& =2\left(\frac{\alpha_{1}^{2 n-1}+\alpha_{2}^{2 n-1}}{2}\right)=2 c_{n} .
\end{aligned}
$$

Theorem 3.2 For any positive integer $n$ we have

$$
c_{n}^{2}=\frac{C_{2 n-1}-1}{2} .
$$


Proof Evaluating $c_{n}^{2}$ using Binet form of Lucas-cobalancing number we get,

$$
\begin{aligned}
c_{n}^{2} & =\left(\frac{\alpha_{1}^{2 n-1}+\alpha_{2}^{2 n-1}}{2}\right)^{2} \\
& =\frac{\alpha_{1}^{2(2 n-1)}+\alpha_{2}^{2(2 n-1)}+2 \alpha_{1}^{2 n-1} \alpha_{2}^{2 n-1}}{4} \\
& =\frac{\alpha_{1}^{2(2 n-1)}+\alpha_{2}^{2(2 n-1)}-2}{4}=\frac{C_{2 n-1}-1}{2} .
\end{aligned}
$$

Corollary 3.1 For any positive integer $n$ we have

(1) $\sum_{i=1}^{n} c_{i}=\frac{C_{n}-1}{2}$

(2) $\sum_{i=1}^{n} c_{i}^{2}=\frac{B_{2 n}-2 n}{4}$.

Proof For (1), consider relation from Theorem 3.1 that is $C_{i}-C_{i-1}=2 c_{i}$ and taking summation from 1 to $n$ we get $C_{n}=2 \sum_{i=1}^{n} c_{i}+1$, by assuming $C_{0}=1$.

Alternatively,

$$
\begin{aligned}
\sum_{i=1}^{n} c_{i} & =\left(\frac{\alpha_{1}^{1}+\alpha_{1}^{3}+\alpha_{1}^{5}+\ldots+\alpha_{1}^{2 n-1}}{2}\right)+\left(\frac{\alpha_{2}^{1}+\alpha_{2}^{3}+\alpha_{2}^{5}+\ldots+\alpha_{2}^{2 n-1}}{2}\right) \\
& =\frac{\alpha_{1}}{2}\left(\frac{\alpha_{1}^{2 n}-1}{\alpha_{1}^{2}-1}\right)+\frac{\alpha_{2}}{2}\left(\frac{1-\alpha_{2}^{2 n}}{1-\alpha_{2}^{2}}\right) .
\end{aligned}
$$

Now simplifying above equation using $\alpha_{1}=-\alpha_{2}^{-1}$, we get

$$
\frac{2\left(\alpha_{1}^{2 n}+\alpha_{2}^{2 n}\right)-4}{8} \text {. }
$$

Therefore, using Binet form of Lucas-cobalancing number, above equation reduces to $\frac{1}{2}\left(C_{n}-1\right)$.

For (2) taking summation from 1 to $n$ for $2 c_{n}^{2}=C_{2 n-1}-1$ in Theorem 3.2 we get,

$$
2 \sum_{i=1}^{n} c_{i}^{2}=\sum_{i=1}^{n} C_{2 n-1}-n .
$$

Now we evaluate $\sum_{i=1}^{n} C_{2 n-1}$,

$$
\begin{aligned}
\sum_{i=1}^{n} C_{2 n-1} & =C_{1}+C_{3}+\ldots+C_{2 n-1} \\
& =\left(\frac{\alpha_{1}^{2}+\alpha_{1}^{6}+\alpha_{1}^{10}+\ldots+\alpha_{1}^{2 n-1}}{2}\right)+\left(\frac{\alpha_{2}^{2}+\alpha_{2}^{6}+\alpha_{2}^{10}+\ldots+\alpha_{2}^{2 n-1}}{2}\right) .
\end{aligned}
$$


Since, the series are in geometric progressions where $\alpha_{1}=1+\sqrt{2}>1$ and $\alpha_{2}=1-\sqrt{2}<1$. So, we get summation of $\sum_{i=1}^{n} C_{2 n-1}$ as

$$
\alpha_{1}^{2}\left(\frac{\alpha_{1}^{4 n}-1}{\alpha_{1}^{4}-1}\right)+\alpha_{2}^{2}\left(\frac{1-\alpha_{2}^{4 n}}{1-\alpha_{2}^{4}}\right) .
$$

Using $\alpha_{1}=-\alpha_{2}^{-1}$ and

$$
B_{n}=\frac{\alpha_{1}^{2 n}-\alpha_{2}^{2 n}}{4 \sqrt{2}}
$$

simplifying the above equation we get $\frac{B_{2 n}}{2}$. So, putting this in equation (4) reduces it to

$$
\frac{B_{2 n}-2 n}{4} \text {. }
$$

This proved our corollary.

Theorem 3.3 For any positive integer $n$ we have

(1) $C_{n-1} C_{n+1}=\frac{C_{2 n}+17}{2}$

(2) $c_{n-1} c_{n+1}=\frac{C_{2 n-1}-17}{2}$

(3) $C_{n-1} C_{n+1}+c_{n-1} c_{n+1}=\frac{C_{2 n}+C_{2 n-1}}{2}$

\section{Proof}

For (1), considering Binet form of Lucas-balancing number and evaluating $C_{n-1} C_{n+1}$,

$$
\left(\frac{\alpha_{1}^{2(n-1)}+\alpha_{2}^{2(n-1)}}{2}\right)\left(\frac{\alpha_{1}^{2(n+1)}+\alpha_{2}^{2(n+1)}}{2}\right) .
$$

Now simplifying above equation using $\alpha_{1}=-\alpha_{2}^{-1}$ we get $\frac{1}{2}\left(C_{2 n}+17\right)$.

For (2), considering Binet form of Lucas-cobalancing number and evaluating $c_{n-1} c_{n+1}$,

$$
\left(\frac{\alpha_{1}^{2 n-3}+\alpha_{2}^{2 n-3}}{2}\right)\left(\frac{\alpha_{1}^{2 n+1}+\alpha_{2}^{2 n+1}}{2}\right) .
$$

Now simplifying the above equation using $\alpha_{1}=-\alpha_{2}^{-1}$, we get $\frac{1}{2}\left(C_{2 n-1}-17\right)$.

For (3), just add (1) and (2).

Theorem 3.4 For any positive integer $n$ we have

(1) $\lim _{n \rightarrow \infty} \frac{C_{n+1}}{C_{n}}=3+2 \sqrt{2}$

(2) $\lim _{n \rightarrow \infty} \frac{c_{n+1}}{c_{n}}=3+2 \sqrt{2}$ 


\section{Proof}

For (1), we consider Binet form of Lucas-balancing number,

$$
\lim _{n \rightarrow \infty} \frac{C_{n+1}}{C_{n}}=\frac{\alpha_{1}^{2(n+1)}+\alpha_{2}^{2(n+1)}}{\alpha_{1}^{2 n}+\alpha_{2}^{2 n}} .
$$

Since $\alpha_{1}>\alpha_{2}$, so dividing above equation by $\alpha_{1}^{2 n}$ we get $\alpha_{1}^{2}=3+2 \sqrt{2}$.

For (2), we consider Binet form of Lucas-cobalancing number,

$$
\lim _{n \rightarrow \infty} \frac{c_{n+1}}{c_{n}}=\frac{\alpha_{1}^{2 n+1}+\alpha_{2}^{2 n+1}}{\alpha_{1}^{2 n-1}+\alpha_{2}^{2 n-1}}
$$

Since $\alpha_{1}>\alpha_{2}$, so dividing above equation by $\alpha_{1}^{2 n}$ we get $\alpha_{1}^{2}=3+2 \sqrt{2}$. This proved our theorem.

Theorem 3.5 For any positive integer $n$ we have

(1) $\sum_{i=1}^{n} C_{i}=\frac{c_{n+1}-1}{2}$

(2) $\sum_{i=1}^{n} C_{i}^{2}=\frac{c_{2(n+1)}+c_{2 n+1}-8+16 n}{32}$

Proof For (1), consider the Binet form of Lucas-balancing number and evaluating $\sum_{i=1}^{n} C_{i}$,

$$
\begin{aligned}
\sum_{i=1}^{n} C_{i} & =\left(\frac{\alpha_{1}^{2}+\alpha_{1}^{4}+\alpha_{1}^{6}+\ldots+\alpha_{1}^{2 n}}{2}\right)+\left(\frac{\alpha_{2}^{2}+\alpha_{2}^{4}+\alpha_{2}^{6}+\ldots+\alpha_{2}^{2 n}}{2}\right) \\
& =\frac{\alpha_{1}^{2}}{2}\left(\frac{\alpha_{1}^{2 n}-1}{\alpha_{1}^{2}-1}\right)+\frac{\alpha_{2}^{2}}{2}\left(\frac{1-\alpha_{2}^{2 n}}{1-\alpha_{2}^{2}}\right) .
\end{aligned}
$$

Simplifying the above equation using $\alpha_{1}=-\alpha_{2}^{-1}$ we get $\frac{1}{2}\left(c_{n+1}-1\right)$.

For (2), we have $C_{i}^{2}=\frac{1}{4}\left(\alpha_{1}^{4 n}+\alpha_{4}^{4 n}+2\right)$, so evaluating $\sum_{i=1}^{n} C_{i}^{2}$

$$
\sum_{i=1}^{n} C_{i}^{2}=\frac{\left(\alpha_{1}^{4}+\alpha_{1}^{8}+\alpha_{1}^{12}+\ldots+\alpha_{1}^{4 n}\right)+\left(\alpha_{2}^{4}+\alpha_{2}^{8}+\alpha_{2}^{12}+\ldots+\alpha_{2}^{4 n}\right)+2 n}{4} .
$$

Simplifying the above equation using Binet form of Lucas-balancing number and $\alpha_{1}=-\alpha_{2}^{-1}$ we get

$$
\frac{C_{2(n+1)}-C_{2 n}-16}{64}+\frac{n}{2} .
$$

Now, by Theorem 3.1 we get $C_{2(n+1)}-C_{2 n+1}=2 c_{2(n+1)}$ and $C_{2 n+1}-C_{2 n}=2 c_{2 n+1}$ which reduces the equation (5) to

$$
\frac{c_{2(n+1)}+c_{2 n+1}-8+16 n}{32} .
$$

This completes the proof. 
Lemma 3.1 $2 c_{n} C_{n-1}=c_{2 n-1}+1, n \in N$.

Proof Considering $c_{n} C_{n-1}$ and evaluating it using Binet form of Lucas-balancing and Lucas-cobalancing number

$$
\begin{aligned}
\frac{\left(\alpha_{1}^{2 n-1}+\alpha_{2}^{2 n-1}\right)\left(\alpha_{1}^{2(n-1)}+\alpha_{2}^{2(n-1)}\right)}{4} & =\frac{\alpha_{1}^{4 n-3}+\alpha_{2}^{4 n-3}+\alpha_{1}^{2 n-1} \alpha_{2}^{2 n-2}+\alpha_{1}^{2 n-1} \alpha_{2}^{2 n-2}}{4} \\
& =\frac{\alpha_{1}^{4 n-3}+\alpha_{2}^{4 n-3}+2}{4}=\frac{c_{2 n-1}+1}{2}
\end{aligned}
$$

This proved the lemma.

Theorem 3.6 For any positive integer n, balancing, Lucas-balancing and Lucas-cobalancing sequence satisfy recurrence relation $C_{n}+c_{n}=4 B_{n}$.

Proof By the definition of Lucas-balancing we get recurrence relation $C_{n}^{2}=8 B_{n}^{2}+1$, now simplifying it using Theorem 3.1 and $C_{n-1}^{2}=8 B_{n-1}^{2}+1$ we get

$$
C_{n-1}^{2}+4 c_{n}^{2}+4 C_{n-1} c_{n}=8 B_{n}^{2}+1 .
$$

Then by using Theorem 3.2, Lemma 3.1 and $B_{2 n-1}=B_{n}^{2}-B_{n-1}^{2}$ (see [7]) the above equation is further simplified to $C_{2 n-1}+c_{2 n-1}=4 B_{2 n-1}$. Replacing $n$ by $\left(\frac{2 n+1}{2}\right)-1$ in previous equation we get $C_{2 n}+c_{2 n}=4 B_{2 n}$. So we can generalize the relation between balancing, Lucas-balancing and Lucas-cobalancing sequence through a recurrence relation $C_{n}+c_{n}=4 B_{n}$. This completes the proof.

\section{Conclusion}

In this paper, we investigate some new properties of Lucas-balancing and Lucas-cobalancing. We connect balancing number, Lucas-balancing and Lucas-coblancing through a recurrence relation.

\section{References}

[1] Behera, A. and Panda, G. K. On the square roots of triangular numbers. Fibonacci Quarterly. 1999. 37(2): 98-105.

[2] P. K. Ray. Balancing and Cobalancing Numbers, http://www.nitrkl.ac.in/, 2009, http://ethesis.nitrkl.ac.in/2750/.

[3] Panda, G. K. and Ray, P. K. Cobalancing Numbers and Cobalancer, International Journal of Mathematics and Mathematical Sciences. 2005. 8: 1189-1200.

[4] Panda, G. K. Some fascinating properties of balancing numbers. in Proc. Eleventh Internat. Conference on Fibonacci Numbers and Their Applications, Cong. Numerantium. 2009. 194: 185-189.

[5] Panda, G. K. and Ray, P. K. Some Links of Balancing and Cobalancing numbers with Pell and Associated Pell Numbers, Bulletin of the Institute of Mathematics Academia Sinica (New Series). 2011. 6: 41-72. 
[6] Lockwood, E. H. A side-light on Pascal's triangle, Math.Gazette. 1967. 51: 243-244.

[7] Ray, P. K. Curious congruences for balancing numbers. Int. J. Contemp. Sciences. 2012. 7: 881-889. 